\section{Acquired Pulmonary Arteriove- nous Malformation in a Case of a Pediatric Adrenocortical Carcinoma}

Sir,

Pulmonary arteriovenous malformations (PAVMs) are abnormal vascular connections, most often between a pulmonary artery and a pulmonary vein; therefore, the blood bypasses the normal pulmonary capillary bed and results in an intrapulmonary right-to-left shunt. Clinically, this may present with hypoxemia and/or complications of pulmonary embolisation. Majority of the cases are hereditary, while a small proportion is idiopathic or secondary. ${ }^{1}$ Most common cause of hereditary PAVM is hereditary hemorrhagic telangiectasia (HHT); while acquired causes may include chest surgery, trauma, actinomycosis, schistosomiasis, hepatic cirrhosis-related hepatopulmonary syndrome (HPS), and metastatic carcinoma. ${ }^{2}$ PAVM was first described in 1897 on autopsy of a 12-year boy. ${ }^{3}$ The first successful case of surgical removal was reported in 1942 by Hepburn and Dauphinee. ${ }^{4}$ Diagnosis of PAVM is often quite challenging. High index of clinical suspicion followed by definitive investigations is required to make the diagnosis. Chest radiography, echocardiography, pulmonary angiography, helical CT scan, magnetic resonance imaging (MRI), and digital subtraction catheter pulmonary angiography (DSCPA), have been used to detect PAVM. ${ }^{1}$ Transthoracic contrast echocardiography (TTCE) is the test of choice for screening PAVM, and if performed alone, has a sensitivity of $98.6 \%$ to detect PAVM. ${ }^{5}$

A3.5-yearfemalechild was diagnosed with adrenocortical carcinoma in January 2019 in Kuwait. At that time, she presented with hypertension and was found to have a large right adrenocortical tumor invading the visceral surface of the liver, with tumor thrombus within inferior vena cava (IVC) extending to the level of right atrium along with a large metastatic thrombus in right pulmonary artery, but no distant metastasis. Mass biopsy revealed adrenocortical carcinoma. As this was an un-resectable tumor, so neo-adjuvant chemotherapy was given, but it did not show any response to chemotherapy. Therefore, the child was put on palliative therapy in April 2019 and presented to us in June 2109 with complaint of progressive bluish discoloration of lips and nails for the past 2 months. Our centre is the largest pediatric hematology/oncology centre in a public sector tertiary care children's hospital with provision of health services free of cost. This patient was being given subcutaneous heparin at that time for the pulmonary thrombus. On examination, the child had central cyanosis but was vitally stable. The heart sounds were normal with no added sounds. Chest auscul- tation showed normal breath sounds. Abdomen was protuberant and a huge ill-defined mass was palpable occupying epigastric, right hypochondrial, umbilical and right lumber regions of abdomen. Pulse oximetry showed saturation of $65 \%$; and inhaled oxygen therapy did not show any improvement in oxygen saturation. This raised the suspicion of arteriovenous (AV) shunting; therefore, echocardiography was done that showed tumor thrombus in the right atrium with no evidence of intra-cardiac shunting. Echocardiography was repeated with bubble contrast, which confirmed the presence of PAVM. We planned to go for computed tomography (CT) - angiography, butthe attendants left against medical advice.

PAVMs are primarily congenital conditions with acquired causes being extremely uncommon. ${ }^{6}$ Intravascular tumor thrombi can arise in various kinds of cancer. The most commonly associated ones are Wilms tumor, renal cell carcinoma, adrenocortical carcinoma and hepatocellular carcinoma. The presence of tumor thrombus has a significant impact over the treatment approach and worsens the prognosis. ${ }^{7}$ There is very scarce data available regarding development of acquired PAVMs in pediatric population. This reported patient is a case of acquired PAVM in pediatric age group with the cause being long-standing pulmonary embolus secondary to tumor thrombus. Though tumor thrombi, associated with adrenal tumors, are a well-known entity but associated pulmonary embolism with development of PAVM is a distinctive presentation.

\section{CONFLICT OF INTEREST:}

The author declared no conflict of interest.

\section{AUTHOR'S CONTRIBUTION:}

Solely contributed to the conception, literature search, writing / revising all drafts of the manuscript and gave final approval as well as agreed to be accountable for all aspects of the manuscript and agreed to act as the corresponding author.

\section{ACKNOWLEDGEMENTS:}

I would like to acknowledge Dr. Jahangir Mustafa (Fellow Pediatric Cardiology) and Dr. Aamnah Zafar Qureshi (Assistant Professor Pediatric Cardiology) for doing the transthoracic echocardiography with bubble contrast and confirming the diagnosis of PAVM.

\section{REFERENCES}

1. Saboo SS, Chamarthy M, Bhalla S, Park H, Sutphin P, Kay $\mathrm{F}$, et al. Pulmonary arteriovenous malformations: Diagnosis. Cardiovasc Diagn Ther 2018; 8(3): 325-37. doi: 10.21037/ cdt.2018.06.01.

2. Cummings KW, Bhalla S. Pulmonary vascular diseases. Clin Chest Med 2015; 36(2):235-48. doi: 10.1016/j.ccm. 2015. 02.007.

3. Churton T. Multiple aneurysms of pulmonary artery. BMJ 1897; i:1223.

4. Hepburn J, Dauphinee JA. Successful removal of hemangioma of lung followed by disapperance of 
polycythemia. Am J Med Sci 1942; 204:681-7.

5. Circo S, Gossage JR. Pulmonary vascular complications of hereditary haemorrhagic telangiectasia. Curr Opin Pulm Med 2014; 20(5):421-8. doi: 10.1097/MCP.000000000 0000076.

6. Gill SS, Roddie ME, Shovlin CL, Jackson JE. Pulmonary arteriovenous malformations and their mimics. Clin Radiol 2015; 70(1):96-110. doi: 10.1016/j.crad.2014. 09.003.

7. Quencer KB, Friedman T, Sheth R. et al. Tumor thrombus: incidence, imaging, prognosis and treatment. Cardiovasc Diagn Ther 2017; 7(Suppl 3):S165-S77.
Rahat-Ul-Ain

Department of Paediatric Hematology/Oncology and Bone Marrow Transplant, The Children's Hospital and Institute of Child Health, Lahore, Pakistan

Correspondence to: Dr. Rahat-Ul-Ain, Department of Paediatric Hematology/Oncology \& Bone Marrow Transplant, The Children's Hospital \& Institute of Child Health, Lahore, Pakistan

E-mail:dr.rkashif@yahoo.com

Received: January 15, 2020; Revised: March 26, 2020; Accepted: April 08, 2020

DOI: https://doi.org/10.29271/jcpsp.2021.01.119 